BENM 2021

International Scientific and Practical Conference "Biotechnology, Ecology, Nature Management"

\title{
ANALYSIS OF THE MICROFLORA OF MUCUS SECRETIONS OF THE WORM (EISENIA FETIDA)
}

\author{
L. I. Buchkova (a)*, A. K. Ponomarev (b), O. G. Bugaev (c), T. I. Horeva (d) \\ *Corresponding author
}

(a) K.G. Razumovsky Moscow State University of Technologies and Management (the First Cossack University), 73, Zemlyanoy Val str., Moscow, Russia, larabychkova@mail.ru

(b) K.G. Razumovsky Moscow State University of Technologies and Management (the First Cossack University), 73, Zemlyanoy Val str., Moscow, Russia, ponomarev777@mail.ru

(c) K.G. Razumovsky Moscow State University of Technologies and Management (the First Cossack University),

73, Zemlyanoy Val str., Moscow, Russia, 12747a473856.22@gmail.com

(d) K.G. Razumovsky Moscow State University of Technologies and Management (the First Cossack University), 73, Zemlyanoy Val str., Moscow, Russia, t.i.horeva@mgutm.ru

\begin{abstract}
In this paper, an experimental analysis of the composition of the microflora of mucus secretions of the California worm (Eisenia fetida)was carried out, when they were cultivated on a mixture of plant residues and rabbit droppings, the relationship of the mucus microflora with the worm itself was revealed under the conditions of the experimental system under consideration, the species composition and percentage ratio of microbial species were established, and the microbial number was determined. It is shown that the microflora retains its properties in the worm mucus for a long time, and does not contain microorganisms that are dangerous to human health. In the framework of this study, no pathogenic genera were found in the mucilage divisions of the California worm; the detected representatives of coli morphic microflora were introduced together with the substrate and stimulated by it. The presence of saprophytic microflora groups involved in decomposition and organic raw materials makes it possible to use worm secretions as a natural reservoir of microflora for enriching the soil with useful microorganisms.
\end{abstract}

2672-8575 ㄷ 2022 Published by European Publisher.

Keywords: Enterobacteria, microbiology, Pseudomonas, reducers, Staphylococcus 


\section{Introduction}

In this paper, the species composition of the symbiotic and other microflora of the California worm (Eisenia fetida) is studied using the example of a specific experimental system. This work is aimed at studying the regularities of the species composition of the mucus microflora of the worm species under consideration in stable conditions of its maintenance, identifying patterns related to the environment, as well as studying the purpose of confirming or refuting the fact of symbiotic relations between the worm and the microflora of its mucus secretions (Domínguez et al., 2010). This work also offers promising opportunities for using the information obtained.

The relevance of this work lies in the innovative application of worms for the differentiation of natural and reductive soil microflora due to the relative cheapness of the process, maintain it in an active form, it is theoretically easier separation of raw materials from worms (collection directly protective mucosal secretions), rather than the cult in the laboratory (which requires some higher level of training at all stages of production), and also because of the possibilities of using this method in low laboratory equipment, which significantly reduces the barrier to entry a little-equipped with the governmental laboratories to further research in this field. In addition to the above, the relevance of this work may be the practical application of the techniques described in this paper, and also, the information obtained by the authors of this article reveals part of the device of the reductive mechanism of the California worm, this information is also valuable.

The theoretical significance of this work lies in the analysis of the species composition of mucus microflora, which has further research benefits in relation to reductive processes, symbiotic relationships, and is also applicable in general, educational and practical microbiology, biology and nematology. The obtained systematic data can be used to describe various symbiotic processes involving worms and microflora, as well as a reference material on similar topics and be an auxiliary material (Sharma et al., 2005).

The practical significance of this work lies in the possibility of immediate application of the experience gained in practice by replanting the California worm under consideration in order to introduce one or another necessary microflora into the soil, correct it or inhibit it to achieve certain results. In addition, the practical value of this work lies in the possibility of using the California worm containment system to cultivate specific microflora for various needs. Also, the experience gained can be applied in practice in the production of cosmetics, medicines, veterinary drugs and other industries where the knowledge of microbiology, nematology and biology in general is somehow applicable (Trigo et al., 1999).

The main difficulties of this work were the difficulties of separating microflora into separate species due to its uniform distribution in the collected volume of mucus secretions, which requires a fairly high level of training of researchers. Thus, without subsequent cultivation of the microflora of mucus collected from worms and subsequent separation of the microflora into pure cultures and its separate cultivation, it is almost impossible to conduct research, however, if the microflora of mucus that is complex in the composition of species also meets the goals of further research, then this aspect is not so important. This point is of great importance in the case that you need a certain representative of is 
sleduetmy microflora of all, or some of them undesirable - which means that slime Californian worm is a complex and difficult-decomposable system and reductive soil microflora, from which with high probability for further silt and finer of its operation it requires treatment, perhaps as a fact - Department of the microflora of mucus and impurities of the environment and the possible pathogenic microflora (Alauzet et al., 2001).

Also, to the problem of this study can be attributed to its narrow focus - details study of the microflora was conducted on creep of only one type of worms, contained in a stable and specific conditions, what should be a logical assumption that the results obtained are specific only under these conditions and in the case and their changes can also be observed changes in the species composition of the microflora (Romanova et al., 2011).

Among other things, California worms are living creatures, and in the case of this disease, changes in the specific and quantitative composition of the mucus microflora, its volumes and other characteristics are also possible, which also requires regular care and monitoring of their health.

\section{Problem Statement}

The problem of this study can be attributed to its narrow focus - details study of the microflora was conducted on creep of only one type of worms, contained in a stable and specific conditions, what should be a logical assumption that the results obtained are specific only under these conditions and in the case often a and their changes can also be observed changes in the species composition of the microflora (Romanova et al., 2011).

\section{Purpose of the Study}

We aimed to subsequent cultivation of the microflora of mucus collected from worms and subsequent separation of the microflora into pure cultures and its separate cultivation, it is almost impossible to conduct research, however, if the microflora of mucus that is complex in the composition of species also meets the goals of further research, then this aspect is not so important.

\section{Research Methods}

Males California worms (Eisenia fetida) were grown under laboratory conditions at the K.G. Razumovsky Moscow State University of Technologies and Management (the First Cossack University).

Vermicontainers with a volume of 20 liters were used for cultivation, in which a ventilation system was provided that provided oxygen access to the substrate from the lower and upper sides of the container, which ensured that the entire volume of the substrate was saturated with oxygen. During cultivation, the substrate temperature was maintained in the range of $20-24^{\circ} \mathrm{C}$, and the substrate humidity was $70-80 \%$ of the total moisture capacity. The $\mathrm{pH}$ value of the substrate ranged from 5.0 to 7.0. The food substrate for obtaining Eisenia fetida biomass was rabbit droppings without additional processing, which was added regularly to vermicontainers as the organic fraction of the droppings was processed by worms. 
The described conditions correspond to the needs of the California worm and (Eisenia fetida) quite qualitatively and meet the criteria of its needs, the worms grown in such conditions are healthy in morphological and physiological characteristics, the presence of diseases or developmental abnormalities was not detected (Sharma et al., 2005).

Based on the fact that this study involves the study of soil and reductive microflora, as well as on the fact that the worms studied are grown in the ground with the addition of a food substrate in the form of rabbit droppings, the following two types of media were determined:

1.Endo-Agar (Agar Endo-TIMING). This nutrient medium was chosen because of its selective properties (suppression of gram-negative microorganisms) and the estimated results of inoculations with this type of media are aimed at determining the presence of a purely group of Enterobacterales microorganisms, which in the framework of this study can indicate the presence of possible pathogenic microflora of this group.;

2.Brian Heart Infusion Agar (Heart agar). This nutrient medium was chosen because ofits high selective properties and also because of its high nutritional properties, which should meet the requirements of the study regarding the selection of the general composition of microflora with the most reliable species composition, the requirements of which are optimally met by this nutrient medium.

Before collecting mucus from the vermicontainer, about 150 grams of live worms were randomly collected in a pre-sterilized plastic container with a sterile instrument to preserve the purity of the bacterial culture.

Further, in laboratory conditions, the mucus was going direct method with worms mechanical pipette Biohit series Proline volume $5 \mu$ land urgent were microbiologically sowing in 5, 10 and 20-fold dilution in sterile $0,9 \% \mathrm{NaCL}$ on a previously prepared Petri bowl with nutrient environments, some of the mucus was collected in a sterile container and placed in a medical refrigerator Liebherr lkexv 1800 with a constant temperature of $+2{ }^{\circ} \mathrm{C}$ for preservation for future research ions, in the case of the need for such.

After microbiological seeding, the petri dishes were placed in the Binder bf 10 thermostat for 7 days (approximately 170 hours) at a constant temperature of $22-24^{\circ} \mathrm{C}$, which was selected based on the approximate temperature of the worm content and the actual temperature of the mucus itself.

Then, after the first day, an auxiliary branch of the study was started - characteristic colonies were selected and transferred to similar Petri dishes with the appropriate type of nutrient media for further breeding of pure crops and their subsequent detailed study for species affiliation. Then, after another day, 37 cultures were tested with them for primary generic differentiation and microscopy, which showed the following results (see Table 1 and 2).

\section{Findings}

Table 1. Results of tests for gender identity

\begin{tabular}{cccccc}
\hline Mobility & Morphology & Gram Test (+l-) & Catalase test(+l-) & Oxidase test (+l-) & Specific genus \\
\hline$+/-$ & rod-shaped & + & + & + & Bacillus \\
+ & rod-shaped & - & + & + & Pseudomonas
\end{tabular}




$\begin{array}{cccccc}- & \text { cocci grouped } & - & - & +/-* & \text { Micrococcus } \\ +/- \text { rod- } & \text { shaped, grouped } & - & + & - & \text { Escherhia } \\ - & \text { cocci grouped } & - & + & - & \text { Acinetobacter }\end{array}$

*The initial results of generic differentiation gave contradictory indications of oxidase in the genus Micrococcus, however, it was later revealed that this difference is explained by different species that tend to have both a positive and negative result.

Table 2. Results of species identification tests

\begin{tabular}{cc}
\hline Gender & Representatives of the genus \\
\hline Bacillus & B. subtilis; B. licheniformis. \\
Pseudomonas & P. fluorescense. \\
Micrococcus & M.luteus, M.roseus. \\
Escherhia & E.cloacae, E.aerogenes E.coli spp \\
Acinetobacter & $-*$ \\
\hline *It was not possible to determine for certain the species belonging to the genus Acinetobacter.
\end{tabular}

*It was not possible to determine for certain the species belonging to the genus Acinetobacter.

Further, after 7 days, quantitative results were obtained (see Table 2, Figure 1): As a result of counting colonies, averaging the result, and comparing various degrees of dilution with each other, it was determined that the mucus of the California worm Eisenia fetida contained under the conditions described earlier is approximately $1 \times 10^{\wedge} 4 \mathrm{CFU} / \mathrm{ML}$.
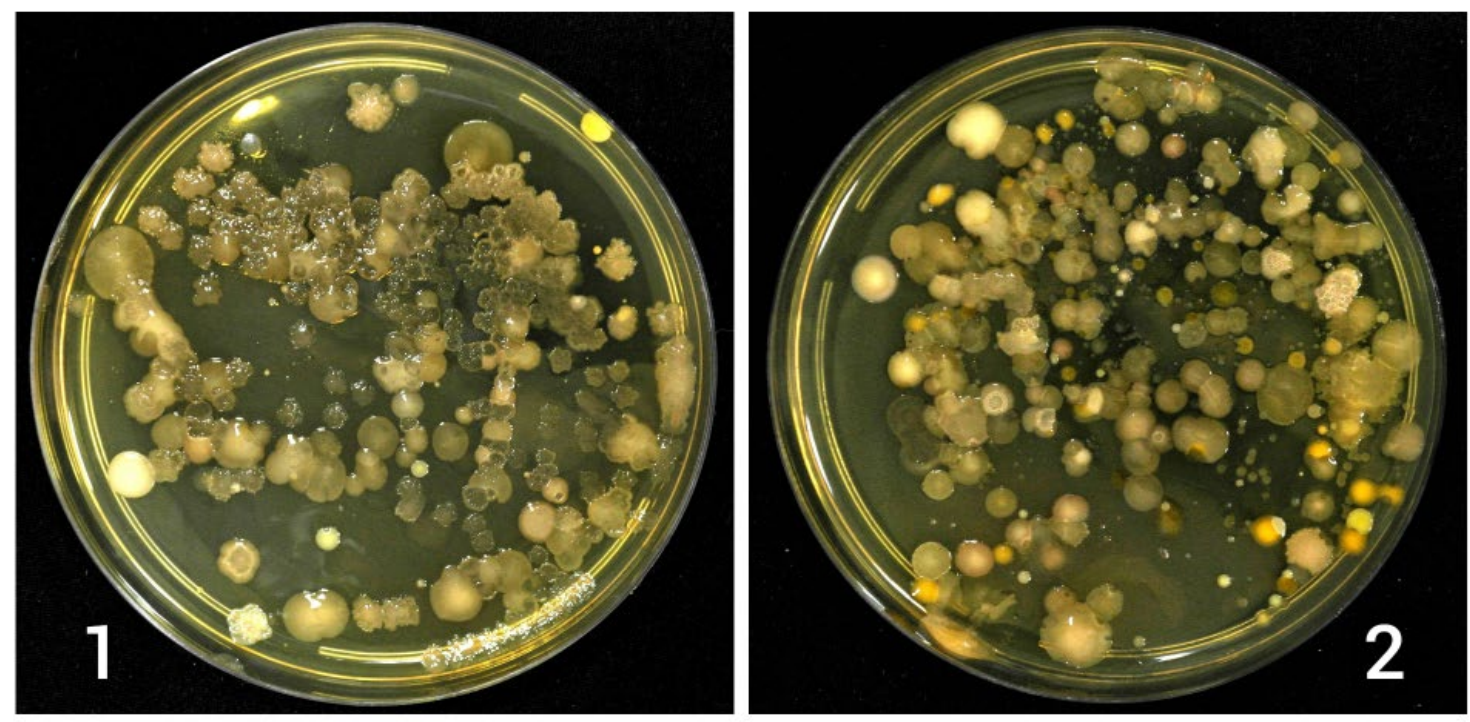

Figure 1. Presentation collage of kolonium on petri dishes with Heart agar, microbiological inoculation 10x dilution period and incubation-7 days $(1 ; 2)$ 


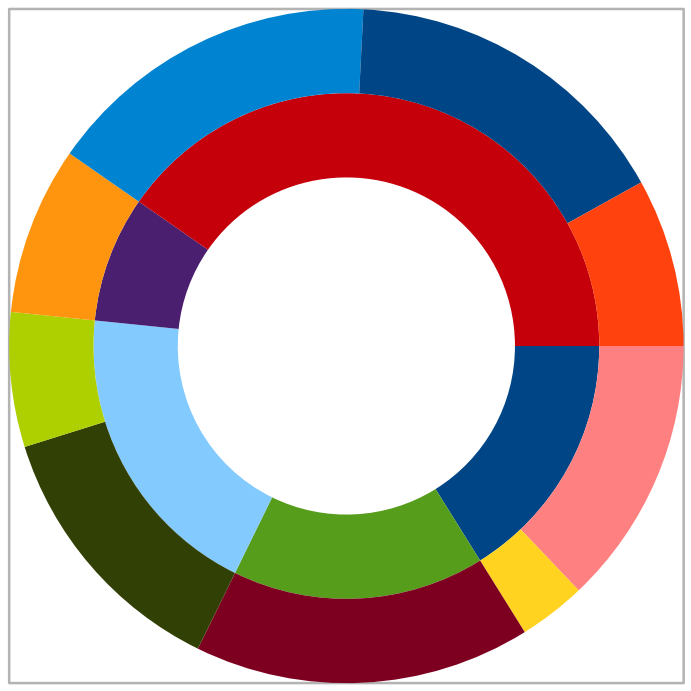

- Bacillus

B. subtilis

B. Llcheniformis

- род Pseudomonas

- P. fluorescense

Micrococcus

- M. luteus

M. roseus

- род Acinetobacter

- Acinetobacter spp

- род Escherhia

- E. cloacae

- E. aerogenes

E. coli spp

Figure 2. Correlation of the species diversity of worm microflora relative to its CFU / ML

Based on the data obtained, it can be argued that the mucus microflora of the California worm Eisenia fetida consists of denitrifying land and colimorphicmicro flora in approximately equal proportions. This point is primarily due to the introduction of rabbit droppings as an organic substrate, which, in addition to soil, is also a carrier substance in vermicontainers.

On the basis of known local data Californicum worm is possible to argue that the composition of the microflora (denitrifying bacteria of the genera Bacillus, Micrococcus, Pseudomonas (see Figure 2)) mucus is symbiotic soil bacteria, within which is California her thus increases its productivity because in addition to its own processing organic in these processes involved specific microflora (Zhabina et al., 2020.).

Analysis directly protective mucosal secretions showed that they have a $\mathrm{pH}$ in the range of zone of 5.5 to 7.0, which also corresponds to the $\mathrm{pH}$ of the substrate in varmintair, and considering its temperature equal to the temperature of the substrate can be assumed that this corresponds to the range of living conditions considered denitrifying microflora, which displays the tons of possible auspiciousness of protective mucosal secretions Californian worm as a kind of "storage" of beneficial microflora. This point was also taken into account when further studying the previously prepared mucus contained in the refrigerator - after approximately 120 hours in separation from the worm and substrate, the secretions retained their microbiological composition and other parameters remained close to the original ones with non-critical changes that can be considered as an error in the study, which can be taken as good preserving qualities of mucus. Also, based on the latter, it can be assumed that these changes occurred due to the activity of the mucus microflora, which did not die under the conditions of separation of the latter from the worm. Among other things, it was found that mucus contains some insignificant amounts of nitrates, nitrites, sugars, and other substances that indicate organic matter reduction processes (Khryukin et al., 2018). These substances can be produced both by the worm itself and by the microflora 
contained in the mucus. It is assumed that these substances can be used both by the California worm itself and by the microflora, which, in the latter case, can confirm the favorability of worm mucus as a special environment for the maintenance of terrestrial microflora and a sign of symbiotic relationships, as a result of which the worm's work is simplified (Edwards \& Burrows, 1988).

Callimorpha microflora, not being typical and dominant for the microbiological composition of mucus Californian worm, most likely, was listed with the organic matter in the form of cualiciamo litter, however, as described earlier, the experimental worms are healthy and do not have abnormalities in the physiological, behavioral, and other characteristics, as can be seen that the microflora malicious worm really not skazais (Tereshchenko et al., 2012). This point is due to its adaptive mechanism to organic matter reduction, high immunity and integrity of the epithelium, which in an initially healthy worm does not allow pathogens to enter its body and reliably protects against diseases. However, the fact of inhibition of colimorphic microflora was also not noticed. As a fact, it should be taken into account that colimorphic microflora, although it is not definitely a beneficial component of the species composition of slime secretions of the California worm, other potentially dangerous microflora agents from other genera were not observed. It is also worth considering the supposed high dependence of the microflora composition on the substrate used, which opens up a large range of more detailed studies of this dependence, based on which it can be reasonably assumed that the colimorphic microflora in the conditions of this particular study could be seriously stimulated by rabbit droppings, which are used as a food substrate (Delgado et al., 1995)

\section{Conclusion}

The mucus of the Coliform worm has a rich microbiological composition ( $\left.1 \times 10^{\wedge} 4 \mathrm{CFU} / \mathrm{ML}\right)$, which, under the described conditions, makes up 4 genera in total with six species of soil microflora and 1 genus of colimorphic microflora with three subspecies found.

The mucus secretions of the Californiachick are able to preserve their microflora and other characteristics for at least 120 hours at a low temperature, on the basis of which it is possible to judge their good preserving properties, which also justifiably indicates the purpose of mucus as an adaptive mechanism necessary for the maintenance of beneficial microflora.

Experimentally, it was found that in the composition of the microflora of the mucus of the California worm there are species of denitrifying microflora, such as B.subtilis; B.licheniformis, M..luteus, M..roseus, P..fluorescense, which contribute to the worm in the production of organic matter and a small number of indeterminate species of the genus Acinetobacter.

Experimentally, it was found that the microflora of the mucus of the California worm contains species of polymorphic microflora, such as E.cloacae, E.aerogenes, and a certain number of other E.coli spp that do not have a harmful effect on the worm, trapped in the mucus with an organic substrate in the form of rabbit droppings.

In the framework of this study, no pathogenic genera were found in the mucilage divisions of the California worm; the detected representatives of coli morphic microflora were introduced together with the substrate and stimulated by it. This study did not reveal active pathogens, however, the results obtained are applicable to the system specifically considered and all similar systems, however, due to the 
assumed high dependence of the microflora of mucus secretions not only on the health of the worm itself, but also on the composition of the substrate in which it lives, which opens up a large range of further research in this area.

\section{References}

Alauzet, N., Roussos, S., Garreau, H., \& Vert, M. (2001). Microflora dynamics in earthworms casts in an artificial soil (biosynthesol) containing lactic acid oligomers. Brazilian Archives of Biology and Technology, 44(2), 113-119. https://doi.org/10.1590/S1516-89132001000200001

Delgado, M., Bigeriego, M., Walter, I., \& Calvo, R. (1995). Use of the California red worm in sewage sludge transformation. Turrialba, 45(1-2), 33-41.

Domínguez, J., Aira, M., \& Gómez-Brandón, M. (2010). Vermicomposting: earthworms enhance the work of microbes. In Microbes at work (pp. 93-114). Springer, Berlin, Heidelberg. https://doi.org/10.1007/978-3-642-04043-6_5

Edwards, C. A., \& Burrows, I. (1988). The potential of earthworm compost as plant growth media. Earthworms in waste and environemtnal Management (pp. 21-32). SPB Academic Press.

Khryukin, N. N., Dedov, A. V., \& Nesmeyanova, M. A. (2018). Dynamics of decomposition of plant residues in typical chernozem. Agrochemical Bulletin, 1.

Romanova, E. M., Mukhitova, M. E., \& Titova, E. V. (2011). General and distinctive features of the microbiocenosis of industrial vermiculture eisenia fetida andrey (bouche, 1972) and its natural analog eisenia fetida (savigny, 1826). Bulletin of the Ulyanovsk State Agricultural Academy, 4 (16).

Sharma, S., Pradhan, K., Satya, S., \& Vasudevan, P. (2005). Potentiality of earthworms for waste management and in other uses-A review. The Journal of American Science, 1(1), 4-16.

Tereshchenko, N. N., Yunusova, T. V., \& Pisarchuk, A. D. (2012). Microorganisms-unique indicators of vermicompost quality. Achievements of Sci. and Technol. in Agroindustrial Complex, 5(58), 17551315.

Trigo, D., Barois, I., Garvin, M. H., Huerta, E., Irisson, S., \& Lavelle, P. (1999). Mutualism between earthworms and soil microflora. Pedobiologia, 43(6), 866-873.

Zhabina, N. A., Mikhaleyko, B. A., \& Chikhireva, V. V. (2020). Review of existing methods of processing organic waste by earthworms. In stimulating the scientific and technical potential of society in the strategic period (pp. 51-58). OMEGA SCIENCE. 